\title{
Etude chimique et toxicologique d'une plante égyptienne: Orobanche minor Sutton
}

\author{
par Shokry H. KAMEL
}

Orobanche minor est connue en Égypte sous les noms de «El Halouk » ou de «Zobb Elkeh »; en divers pays, elle a reçu les appellations suivantes « Clover broom-rape ", Epifagus, Epiphegus, Epifagus virginiae (L.) Bart., Leptamnium virgi-

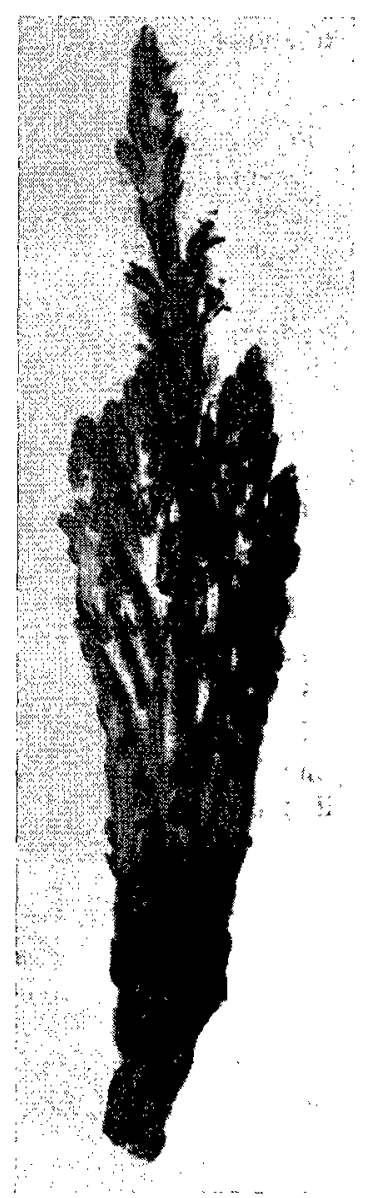

nianum (L.) Raff., "Beech-drops ", " Cancer root», Aphyllon uniflorum, Conopholis americana, "Orobanche de Virginie 》 et "Krebswurz ».

De couleur pourpre, mesurant environ $30 \mathrm{~cm}$ de haut, elle porte une inflorescence en épi lâche dont les principales caractéristiques peuvent être résumées somme suit : sépales nombreux, ovales, acuminés, veinés; corolle blanche ou: parfois jaunâtre: étamines poilues dans la partie inférieure, lisses au-dessus; style presque tout à fait lisse; stigmate pourpre.

Wahby (1940) signale que la periode de floraison de l'Orobanche se situe de janvier à mai; selon Johns (1902), elle est plus tardive (juin-juillet). Quant à nous, nous avons eu l'occasion de récolter cette plante en floraison à partir du début de novembre.

Du point de vue organoleptique, il convient de noter que $1^{\prime} O$. minor a un goût amer, astringent, qui diminue après dessiccation.

L'aire géographique de cette plante est relativement vaste. Muencher (1948) signale qu'O. minor est largement répandue dans toute l'Europe, d'où elle a été introduite aux États-Unis. Elle s'y est propagee dans toute la partie atiantique, du New Jersey à la Caroline du Nord et plus rarement vers l'ouest. En Égypte, on la trouve communément dans la vallée et le delta du Nil. Elle est extrêmement répandue dans les prairies de berseem (trèfle égyptien), qui constitue le principal fourrage distribué en Égypte aux animaux, ainsi que dans les champs de diverses plantes cultivées. On peut donc attribuer à l'O minor un rôle néfaste, non seulement par son action sur les récoltes, mais encore par sa nocivité pour les animaux domestiques. C'est ainsi que Wahby (1940) a signalé que les animaux qui ont consommé du berseem renfermant une forte proportion $d^{\prime} O$. minor sont atteints de violentes coliques tandis que Long, dès 1927, estimait que l'O. minor, en raison de ses propriétés nocives, devrait être l'objet d'investigations.

Par contre, il faut aussi noter que cette plante semble dotée de quelques vertus médicinales. Elle est notamment utilisée comme diurétique, laxatif et antidiabétique (Tazkaret Ibn Dawood).

D'autre part, Wood (1926) a signalé qu' on l'utilise par la voie interne contre les affections intestinales, mais que sa grande renommée dans la médecine populaire vient de ce que l'on croit à son efficacité 
contre les ulcérations chroniques à caractère cancéreux sur lesquelles on l'utilise en applications directes.

Pour toutes ces raisons, il nous a paru nécessaire de soumettre l'O. minor à quelques investigations visant à préciser la nature des principes actifs, leur degré de toxicité pour certains animaux domestiques ou de laboratoire, enfin leur éventuelle activité pharmacodynamique. La plante fleurie utilisée pour ces recherches a été récoltée dans des champs de choux-fleurs et de choux massivomont infostés, à Moderiet El Giza (Égypte), au cours des mois de novembre et décembre.

\section{I. - ANALYSE DE LA PLANTE ET ISOLEMENT DES PRINCIPES ACTIFS}

\section{A. - Examen préliminaire.}

Après dessiccation totale à l'air libre, la plante broyée finement fournit une poudre qui, par extraction à l'eau bouillante, donne un filtrat présentant les caractéristiques suivantes :

Io Coloration brun-chocolat, réaction légèrement acide au papier de tournesol.

$2^{\circ}$ Après addition au filtrat d'une solution de chlorure ferrique, on observe la formation d'un précipité verdâtre sale, tandis qu'après addition d'acétate de plomb, il se forme un précipité floconneux, jaunâtre, indiquant la présence de tanin.

$3^{\circ}$ La recherche de substances réductrices (sucres réducteurs, glucosides et saponines) a été effectuée comme suit :

a) Sucres réducteurs. - Avec les réactifs de Fehling et de Benedict, on obtient un précipité rouge d'oxyde de cuivre signalant la présence de sucres.

b) Substances glucosidiques. - Après hydrolyse et alcalinisation légère à l'aide de soude caustique, le filtrat réduit les réactifs de Fehling et de Benedict de' façon plus nette qu'avant l'hydrolyse, ce qui indique la présence de glucosides.

c) Saponines. - L'addition au filtrat d'une solution de chlorure mercurique provoque la formation d'un précipité blanc. Un miroir d'argent métallique se forme lorsqu'on laisse séjourner au bain-marie bouillant, pendant environ 10 minutes, un mélange à parties égales de filtrat et de solution de nitrate d'argent ammoniacal. Ces deux tests indiquent la présence de saponines.

$4^{\circ}$ Le filtrat acidifié et refroidi, soumis à l'action des réactifs de Mayer et de Wagner donne, dans les deux cas, un faible précipité qui suggère la présence d'alcaloïdes, bien que certains glucosides puissent donner des réactions semblables avec ces deux réactifs (Fahmy, 1933). De ce fait, on a jugé nécessaire de tenter d'isoler les alcaloïdes éventuels.

$5^{\circ}$ Le filtrat obtenu après extraction de la poudre végétale par l'eau froide a été soumis aux tests suivants :

a) Recherches des oxydases, peroxydases et amylases : résultat négatif.

b) Recherche d'amidon : résultat positif, l'addition au filtrat d'une solution iodo-iodurée fait apparaître une coloration bleue.

$6^{\circ}$ Le filtrat obtcnu après extraction par l'alcool à $95^{\circ}$ bouillant, donne, après addition d'eau distillée, un précipité jaunâtre indiquant la présence d'une résine.

En résumé, ces tests préliminaires indiquaient la présence de résine, de glucosides et perıt-être d'alcaloïdes dans l'O. minor.

\section{B. - Séparation des principes actifs.}

$1^{0}$ Glucosides. - $100 \mathrm{~g}$ de poudre de plante sèche ont été soumis à l'extraction par l'eau distillée bouillante, à plusieurs reprises, jusqu'à épuisement. Pour prévenir une hydrolyse possible des substances glucosidiques dans le mélange à la faveur d'une ébullition prolongée en milieu acide, on eut recours à l'addition de carbonate de calcium. Le mélange chaud fut filtré d'abord sur gaze puis sur papier filtre. Les filtrats provenant des diverses extractions furent réunis, puis filtrés à nouveau, pour les clarifier le plus possible. Le filtrat clair fut alors traité par la solution d'acétate de plomio afin d'éliminer le tanin sous forme de tannate de plomb insoluble.

Le nouveau filtrat fut soumis à des traitements successifs par cette solution d'acétate de plomb jusqu'à ce qu'il n'y eût plus aucun précipité. L'acétate de plomb en excès fut éliminé par action d'un courant d'hydrogène sulfuré; un barbotage final d'air dans le filtrat permit d'en chasser enfin l'hydrogène sulfuré en excès.

Le filtrat obtenu après toutes ces opérations fut évaporé sous vide jusqu'à siccité. Le résidu, repris dans une quantité aussi faible que possible d'alcool absolu bouillant, fut filtré à chaud, puis refroidi à la glacière.

La purification fut poussée aussi loin que possible en faisant dissoudre le résidu solide brut obtenu après évaporation de la solution précédente, dans de l'alcool absolu et en additionnant cette seconde solution d'une quantité d'éther suffisante poür provoquer un trouble abondant. On laissa décanter ce mélange à la glacière pendant 24 heures.

Le liquide surnageant fut alors éliminé et le résidu glucosidique mis à sécher pour obtenir sa cristallisation. 
Les cristaux furent recueillis et pesés. Au cours de plusieurs extractions, leur masse représentait de $0,96 \%$ à $1,2 \%$ de celle de la poudre végétale.

Ces cristaux se présentaient sous la forme de petits prismes agglomérés, en forme d'aiguilles, ressemblant à ceux de l'orobanchine, glucoside extrait de l'O. rapum par Bridel et Charaux (1924).

Aisément solubles dans les alcalis, ils donnent avec cux une coloration orangée.

L'addition d'une solution de chlorure ferrique donne, au contraire, une coloration verte, tandis qu'un précipité jaune-citron se forme sous l'action d'une solution d'acétate de plomb.

$2^{\circ}$ Résine. - $10 \mathrm{~g}$ d'extrait alcoolique d'O. minor furent dissous dans la plus petite quantité possible d'alcool absolu au bain-marie bouillant; la résine fut précipitée par addition à cette solution d'une quantité d'eau distillée légèrement acidifiée par l'acide chlorhydrique, le mélange étant laissé au repos pendant 24 heures. Après décantation du liquide surnageant, le résidu résineux fut lavé à l'eau distillée pour le débarrasser de ses impuretés.

Pesée après dessiccation à $105^{\circ} \mathrm{C}$ au four à air chaud, la résine représentait $36 \%$ de la masse de l'extrait.

$3^{\circ}$ Alcaloïdes. - $10 \mathrm{~g}$ d'extrait alcoolique furent traités plusieurs fois par l'eau distillée acidulée, chacune de ces opérations étant suivie d'une filtration, jusqu'à épuisement total. Les filtrats furent mélangés et vigoureusement agités en présence de chloroforme, pour en extraire les impuretés et spécialement les résines. Ce mélange fut laissé au repos jusqu'à séparation du chloroforme, renfermant les matières colorantes, que l'on élimina. Cette opération fut reṇouvelée juṣqu'à élimination apparente de toutes les impuretés. La solution acide fut alors neutralisée puis rendue légèrement alcaline par l'ammoniaque et soumise à une série d'extractions par le chloroforme. Les extraits chloroformiques obtenus furent réunis et lavés à plusieurs reprises à l'eau distillée, l'eau fut ensuite éliminée. L'addition de sulfate dé soude anhydre permit de débarrasser l'extrait chloroformique des dernières traces deau avant filtration. Le filtrat fut alors évaporé au bainmarie, pour obtenir le résidu sec. Celui-ci était alors très peu important et, soumis à l'action des réactifs de Mayer et de Wagner, ne donnait aucune des réactions indiquant la présence d'une substance alcaloïdique. Ce résidu n'était donc probablement constitué que d'impuretés qui, malgré les précautions prises, avaient échappé aux opérations visant à les éliminer.

\section{C. - Analyse approximative.}

$100 \mathrm{~g}$ de plante sèche finement pulvérisée furent soumis dans un appareil de Soxhlet à des extractions succossivcs par l'éther de pétrole, l'éther, le chloroforme et l'alcool à $95^{\circ}$.

Le résidu de ces opérations fut à son tour soumis à des extractions répétées à l'eau distillée, jusqu'à épuisement; il fut alors soumis successivement à l'action de l'eau bouillante, à celle d'une solution aqueuse d'acide chlorhydrique à $1 \%$ et, après lavage à l'eau, à une extraction par une solution aqueuse de potasse caustique à $1 \%$, On termina par des lavages à l'eau, à l'alcool et à l'éther et par une dessiccation suivie de pesée.

Apres calcination du résidu, on calcula le poids de cellulose brute, par différence entre le poids des cendres et celui du résidu sec.

Les taux suivants d'extrait sec ont été obtenus avec les divers solvants :

Éther de pétrole .................. 0,81

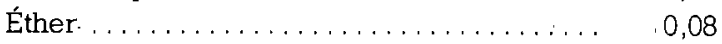

Chloroforme..................... 0,07

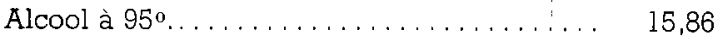

Eau froide....................... 13,25

Eau chaude. . . . . . . . . . . . . . . . 4,90

Eau acidifiée $(\mathrm{HCl} 1 \%$. . . . . . . . . . . . 1,29

Eau alcalinisée $(\mathrm{KOH} 1$ \%) . . . . . . . . . 3,55

$\begin{array}{lr}\text { Résidu final } \ldots \ldots \ldots \ldots \ldots & 48,54 \\ \text { Cendres... . . . . . . . . } & 1,56 \\ \text { Cellulose (par différence) . } & 46,98\end{array}$

\section{Caractères des extraits.}

a) Extrait obtenu par l'éther de pétrole; : cet extrait, de consistance graisseuse, de coloration vert jaunâtre, sans amertume, est furmé prinicipalement de graisses mais contient aussi de la résine et du tanin.

b) L'extrait éthéré est de couleur brun jaunâtre, de consistance graisseuse et possède une saveur âcre et amère. Il contient de la résine.

c) L extrait chloroformique, de couleur brune, est formé de résine et de tanin.

d) L'extrait alcoolique est une masse collante, brun sombre, de goût à la fois amer, astringent et salé, d'odeur herbacée. Il est difficilement soluble dans l'eau froide mais facilement dissoús par I'eau bouillante. Il est formé principalement, de résine, de sucres et de tanin.

e) Les extraits aqueux ont un goût amer et une coloration brun foncé. L'extrait obtenu par l'action de l'eau froide est très collant. Il est fórmé principalement de tanin, de mucilage, d'amidon et: de protéine.

f) Les extraits obtenus par actıon d'eau acidulée ou alcalinisée sont de couleur brune et renferment du tanin.

En résumé, l'étude chimique permet de conclure que la plante verte renferme environ: $1,44 \%$ de 
résine et $0,25 \%$ de substances glucosidiques, qu'elle est riche en tanin et contient de petites quantités de sucre, mais ne renferme pas d'alcaloïdes.

$A$ priori, on peut, penser que l'O. minor doit surtout ses propriétés laxatives à sa forte teneur en résine. L'étude toxicologique permet de confirmer cette hypothèse et de préciser le rôle respectif de la résine et du glucoside dans l'intoxication.

\section{II. - ÉTUDE TOXICOLOGIQUE}

Nos recherches toxicologiques ont été effectuées sur les animaux des espèces suivarıles : chèvres, chiens, rats blancs, crapauds.

La plante ou ses extraits ont été administrés per os, ou par injections intrapéritonéales, intraveineuses et sous-cutanées. Les résultats obtenus permettent d'abord d'affirmer qu'il existe des différences dans la sensibilité des diverses espèces animales à l'intoxication par l'Orobanche.

a) Chèvres. - Quatre chèvres, âgées d'environ un an, furent soumises à une diète préalable de 24 heures. Deux d'entre elles servirent de témoins et reçurent une ration formée uniquement de berseem sans Orobanche. Les deux autres reçurent une ration formée de trois parties d'Orobanche pour une partie de berseem.

Ces dernières consommèrent bien leur ration. Le lendemain, elles présentèrent une diminution de l'appétit et de la rumination, une soif intense et des coliques douloureuses qui allèrent croissant; le troisième jour, les animaux présentèrent des vomissements répétés, de la tristesse, de l'abattement, A la fin du quatrieme jour, l'état d'une chèvre empira, l'animal mourut et fut autopsié ; la deuxième chèvre succomba le lendemain et fut également autopsiée. A ce moment, l'état de santé des animaux témoins était tout à fait normal.

b) Chiens. - L'administration per os à des chiens, de quantités variables d'extrait sec alcoolique, renfermant de la résine, provoqua l'apparition de symptômes d'intoxication. La gravité des troubles variait en fonction de la quantité d'extrait ingérée et de celle qui avait pu être rejetée par vomissements. On n'a toutefois observé aucun cas d'intoxication mortelle après ingestion de l'extrait. L'injection intraveineuse de l'extrait (débarrassé de la résine) permit, par contre, d'obtenir une intoxication mortelle dans un délai de 24 heures. La dose de $12 \mathrm{~g}$ d'extrait par kilo de poids vif se montra régulièrement. mortelle.

c) Rats blancs. - Ils parurent sensibles à l'intoxication mais des doses allant jusqu'à $6 \mathrm{~g}$ d'extrait sec alcoolique par kilo de poids vif, en solution dans l'eau distillée et injectée par voie intrapéritonéale, ne provoquèrent pas la mort.

d) Crapauds. - Ces animaux se montrèrent très résistants. C'est seulement en leur injectant, par voie sous-cutanée, une dose de $20 \mathrm{~g}$ d'extrait sec alcoolique par kilo de poids vif que l'on put obtenir en 24 heures une mortalité de $8 \%$.

Détermination de la Dose Minima Léthale d'extrait alcoolique sec.

a) Chez les rats blancs. - Les essais ont été effectués sur 90 rats des deux sexes, âgés de quatre mois et pesant en moyenne $200 \mathrm{~g}$, répartis en neuf groupes, auxquels on injecta par voie intraperitonéale des doses d'extrait sec (débarrassé de la résine) allant de 0,5 à $1,3 \mathrm{~g}$ par kilo de poids vif.

N.B. - La solution injectable fut obtenue en filtrant une solution d'extrait $\mathrm{sec}$ alcoolique dans de l'eau distillée bouillante.

Les résultats obtenus sont présentés dans le tableau ci-contre.

D'après les résultats présentés dans ce tableau, on a pu établir un graphique en portant en abscisses les logarithmes des doses et en ordonnées les valeurs obtenues par la méthode de la " probit value ».



D'après le graphique, on peut calculer que la D.M.L. est de $9 \mathrm{~g}$ par kilo de poids vif. Ceci est confirmé par les résultats obtenus dans le groupe $\mathrm{V}$ où $50 \%$ des animaux moururent dans les 24 heures qui suivirent l'injection intrapéritonéale de $0,9 \mathrm{~g}$ d'extrait par $100 \mathrm{~g}$ de poids vif, tandis que l'autre moitié du groupe survécut jusqu'à la fin de l'expérience.

b) Chez les chiens. - On a constaté que, par voie endoveineuse, la D.M.L. d'extrait sec alcoolique est de $8 \mathrm{~g}$ par kilo de poids vif. Quand l'extrait, non débarrassé de sa résine, est administré per os, on n'obtient pas d'intoxication mortelle même en utilisant de fortes do'ses. 


\begin{tabular}{|c|c|c|c|c|c|c|c|c|c|c|c|}
\hline \multirow{3}{*}{ GROUPES } & \multirow{3}{*}{$\begin{array}{c}\text { DOSE } \\
\text { d'extrait sec } \\
\text { alcoolique } \\
\text { par } 100 \mathrm{~g} \\
\text { de poids vif }\end{array}$} & & \multicolumn{7}{|c|}{ TEMPS EN HEURES APRES ADMINISTRATION } & \multirow{3}{*}{$\begin{array}{l}\text { NOMBRE } \\
\text { total de morts } \\
\text { après } 24 \mathrm{~h}\end{array}$} & \multirow{3}{*}{$\begin{array}{c}\text { POURCENTAGE } \\
\text { total. } \\
\text { de mortalité }\end{array}$} \\
\hline & & & 1 & 2 & 3 & 4 & 5 & 6 & 7 & & \\
\hline & & \multicolumn{8}{|c|}{ Nombre de rats moris sur 10} & & \\
\hline I & $0,5 \cdot \mathrm{g}$ & & - & - & - & - & - & - & - & - & $0 \%$ \\
\hline II & $0,6 \mathrm{~g}$ & & - & - & - & - & - & - & - & - & $0 \%$ \\
\hline III & $0,7 \mathrm{~g}$ & $:$ & - & - & - & - & 1 & - & 1 & 2 & $20 \%$ \\
\hline IV & $0,8 \mathrm{~g}$ & & - & - & - & - & 1 & 2 & - & 3 & $30 \%$ \\
\hline $\mathrm{V}$ & $0,9 \mathrm{~g}$ & & - & - & 2 & 1 & - & 1 & 1 & 5 & $50 \%$ \\
\hline VI & $1,0 \mathrm{~g}$ & & - & - & 1 & - & 3 & 1 & 2 & 7 & $70 \%$ \\
\hline - VII & $1,1 \mathrm{~g}$ & & - & 3 & 1 & 1 & - & 2 & 1 & 8 & $80 \%$ \\
\hline VIII & $1,2 \mathrm{~g}$ & & 5 & 1 & 2 & 2 & - & - & - & 10 & $100 \%$ \\
\hline IX & $1,3 \mathrm{~g}$ & & 4 & 5 & 1 & - & - & - & - & 10 & $100 \%$ \\
\hline
\end{tabular}

c) Chez les crapauds. - Des doses variables d'extrait alcoolique sans résine furent injectées, dans le sac lymptratique ventral, à des crapauds répartis en plusieurs groupes. On put constater ainsi que la D.M.L. est pour ces animaux de $24 \mathrm{~g}$ par kilo de poids vif.

\section{Constatations nécropsiques.}

L'autopsie des animaux intoxiqués montre que les viscères et surtout le foie, le cœur et les reins sont le siège d'une vive inflammation, avec pétéchies à leur surfaçe. Le cceur est dilaté. Le tractus digestif est congestionné, notamment l'estomac, dont la muqueuse présente de petites taches hémorragiques.

\section{Effets des divers principes actifs.}

Expérimentalement, on a constaté que la résine provoque chez les chiens et les chères des symptômes de gastro-entérite, nausees, soif, vomissements et diarrhée.

La substance glucosidique s'est montrée capable de provoquer chez les animaux d'expérience de l'abattement avec impossibilité de se mouvoír; par ailleurs, l'étude expérimentale a montré que le glucoside est vasodilatateur, déprime le muscle cardiaque et provoque le relâchement des muscles lisses intestinaux.

\section{Causes de la mort.}

En tenant compte des signes cliniques et des lésions, on peut considérer comme causes principales de la mort les troubles digestifs et circulatoires résultant de l'action combinée des divers principes toxiques de l'Orobanche.

\section{III. - ÉTUDE DES PROPRIÉTÉS DIURÉTIQUES DE LA PLANTE}

Quatre rats blancs de $200 \mathrm{~g}$, privés d'eau et d'aliments pendant la nuit précédant le début de l'expérience, reçurent une injection intrapéritonéale de $2 \mathrm{~cm}^{3}$ d'eau distillée et furent placés dans des cages spéciales permettant de recueillir intégralement leur urine dans un cylindre gradué: On évalua ainsi à $18,5 \mathrm{~cm}^{3}$ en moyenne la quantité d'urine excrétée en 24 heures par un rat. Les animaux-furent alors laissés au repos pendant 2 jours, avec un régime normal, puis privés d'eau comme précédemment, et enfin soumis à l'expérience suivante : chaque animal reçut une injection intrapéritonéale d'une dose de $0,5 \mathrm{~g}$ d'extrait sec alcoolique (débarrassé de la résine) par $100 \mathrm{~g}$ de poids vif, dissous dans $2 \mathrm{~cm}^{3}$ d'eau distillée. L'urine fut recueillie et mesurée et l'on constata qu'en 24 heures, chaque rat avait excrété en moyenne $30,5 \mathrm{~cm}^{3}$ d'urine. Ces expériences, répétées plusieurs fois sur de nouveaux animaux, ont donné constamment des résultats identiques. On peut donc conclure que l'Orobanche minor Sutton a vraiment des propriétés diurétiques; il est très probable que ces propriétés soient dues à la présence du glucoside que nous àvons décelé dans l'Orobanche.

\section{CONCLUSION}

De l'ensemble des expériences précédentes, on peut conclure que la plante égyptienne Orobanche minor Sutton est un toxique irritant pour les animaux 
de laboratoire et les animaux domestiques. Ceci confirme les opinions de Wahby et de Long. L'action toxique se manifeste par des symptômes de gastroenterite; elle peut être attribuée à la présence d'une résine et d'un glucoside. Les constatations nécropsiques confirment l'existence d'une gastro-entérite.

Nous avons aussi montré que le principe actif glucosidique est vasodilatateur, exerce une action déprimante sur le muscle cardiaque et provoque le relâchement des muscles lisses intestinaux.

Ces diverses propriétés des principes actifs expliquent l'effet diurétique et purgatif de la plante sur les animauxx. On a déjà dit que la substance glucosidique est vraisemblablement responsable de l'action diurétique tandis que l'action purgative devrait 'être attribuée plus spécialement à la résine.

Faculté de Médecine Vétérinaire, Giza (Égypte).

\section{BIBLIOGRAPHIE}

FAHMY (I.-R.) (1933). - Constituents of Plants and Crude Drugs. Paul Barby, Cairo, Ist ed., p. 58. JOHNS (C.-A.) (1902), - Flowers of the Field. Society for promoting Christian Knowledge, 30 th ed., London, p. 556.
LONG (H.-C.) (1927). - Poisonous Plants of the Farm. Ministry of Agriculture and Fisheries, lst ed., London, p. 52.

MAHDI (M.) et FAHMY(I :-R.) (1934), - Orobanche ramosa. Report of the Pharmaceutical Society of Egypt, Cairo, 5th Year, no VI, p. 35-40.

MUENSCHER (W.-C.) (1948). - Weeds. Macmillan and Co, New York, lst ed., p. 428.

SHARAF (A.) (1949). - Chemical Investigation of the Egyptian Plant Euphorbia peblus. The British Vet. J., vol. 105, no 4, p. 128-135.

SHARAF (M.) (1926). — An English-Arabie Dictionnary. Ministry of Education, Egypt, lst ed., p. 181 et 577 .

SHIHATA (I.-M.) (1953). - Botanical and Chemical Investigation of the Egyptian Plant Anagallis arvensis. The Egyptian Vet. Med. J., vol. I no 1, p. 53-63.

WAHBY (A.-M.) (1940), - Egyptian Plants Poisonous to Animals. El Horia Press, Cairo, lst ed., p. 24.

WOOD (C.) (1926). - The Dispensatory of U.S.A. Lippincott Co, 2 st ed., p. 1292.

\section{SUMMARY}

A plant, Orobanche minor Sutton, which is very widespread in Egypt, and known as El Halouk in the vernacular, when consumed in large quantities by cattle, causes some intoxication with signs of varying degrees of gastro-enteritis.

Analysis of this plant has demonstrated the nature of its main contents and results of feeding experiments suggest that its toxic effects may be caused by a resin and a glucoside. The latter constituent produces an intense vasodilatation which could explain the diuretic as well as the laxative effects of the plant. These reaction occured in goats, dogs, white rats and toads.

The sensitivity of these animals to the toxic effects of the plant varies with the species. Goats are very sensitive and die after showing signs of acute gastro-enteritis; the effects are less serious in dogs and very mild in rats. Toads are very resistant.

This plant enjoys prestige amongst Africans as it is said to be able to cure some intestinal conditions, and has also been used as a diuretic, laxative and antidiabetic in human therapy.

\section{RESUMEN}

Una planta muy extendida en Egipto, la Orobanche minor Sutton, localmente llamada El Halouk, provoca en el ganado, cuando es consumida en abundancia, una intoxicación que se traduce por sintomas de gastroenteritis mas o menos pronunciada.

El estudio anahtico de esta planta ha permitido determinar sus principales constituyentes y las investigaciones experimentales han demostrado que la accion tóxíca podría atribuirse a la existencia de una resina y de un glucósido. Este ûltimo posée une accion vaso-dilatadora marcada susceptible de explicar los efectos diuréticos y laxativos de la planta, efectos que han sido puestos en evidencia con ocasion de investigaciones toxicológicas experimentales efectuadas en cabras, perros, ratas blancas y sapos.

Conviene destacar la distinta sensibilidad de estos animales a la accion tóxica de la planta. Las cabras son particularmente sensibles y mueren mostrando sintomas de gastro-enteritis aguda; la gravedad de los trastornos es menor en el perro, poco acusada en la rata. El sapo es muy resistente.

Debe señalarse igualmente que esta planta goza, según la población, de propiedades curativas con respecto a ciertas afecciones intestinales. Se emplea asimismo como diurética, laxante y antidiabética en medicina humana. 